\title{
A Low-Reflectivity Vacuum Window for Rectangular Hollow Waveguides
}

\author{
Christian Koenen, Student Member, IEEE, Gerhard F. Hamberger, Student Member, IEEE, \\ Uwe Siart, Member, IEEE, Thomas F. Eibert, Senior Member, IEEE, Hans-Ulrich Nickel, Member, IEEE, \\ Garrard D. Conway, and Ulrich Stroth
}

\begin{abstract}
The design and performance of a broadband and low-reflectivity millimeter-wave vacuum window for rectangular hollow waveguides is presented. A reflectivity below $-15 \mathrm{~dB}$ is achieved over almost the full $W$-band. The vacuum window is sufficiently compact so that two separate channels fit into a single ConFlat CF-35 vacuum flange. The proposed vacuum window utilizes a well-known circuit concept based on two quadrature hybrid couplers together with two identical vacuum barriers (similar to a balanced amplifier). Due to the dielectric discontinuity at the vacuum barrier, a part of the incident wave is transmitted and another part is reflected. A hybrid lattice network recombines the transmitted signals and reroutes all reflected power to absorbers. The proposed low-reflectivity vacuum window has been fabricated in WR10 waveguide technology. Broadband branch-guide couplers enable an operational frequency range of $75 \mathrm{GHz}$ to $105 \mathrm{GHz}$. Ultra-high vacuum compatibility is verified by a helium leak rate test and electrical measurements show good agreement with full-wave simulations.
\end{abstract}

Index Terms-millimeter-wave passive components, millimeterwave circuits, vacuum windows, millimeter-wave devices, waveguide components, balanced vacuum window

\section{INTRODUCTION}

$\mathbf{I}$ $\mathrm{N}$ nuclear fusion experiments, microwave and millimeterwave signals often have to pass a barrier between the external atmosphere and the ultra-high vacuum inside the device to connect the measurement equipment with its frontend. In this work, focus is on the Doppler reflectometry diagnostics in the nuclear fusion experiment ASDEX Upgrade tokamak in Garching, Germany [1], [2], [3, pp. 346]. The Doppler reflectometer operates over almost the whole $W$-band (from $75 \mathrm{GHz}$ to $105 \mathrm{GHz}$ ) and only a rather small CF-35 (ConFlat with $35 \mathrm{~mm}$ tube inner diameter) vacuum flange is available. The connecting hollow waveguides are normally either fundamental-mode rectangular when installation space is restricted or oversized circular hollow waveguides because of their lower losses. The vacuum barrier is realized by a

Manuscript received Month dd, yyyy; revised Month dd, yyyy.

This work was supported by the Helmholtz Association of German Research Centers within the framework of the Helmholtz Virtual Institute on "Plasma Dynamical Processes and Turbulence Studies using Advanced Microwave Diagnostics".

C. Koenen, G. F. Hamberger, U. Siart, and T. F. Eibert are with the Chair of High-Frequency Engineering, Department of Electrical and Computer Engineering, Technical University of Munich, 80290 München, Germany

H.-U. Nickel is with the Product Development Department, Spinner GmbH, 83620 Feldkirchen-Westerham, Germany

G. D. Conway and U. Stroth are with the Max-Planck-Institute for Plasma Physics, 85748 Garching, Germany dielectric disk which should be transparent to the guided wave while featuring a sufficiently low gas leakage. If the dielectric constant of the disk differs from that of the waveguide filling, reflections occur that may lead to distortions or even damage in the transmitter. In case of a vacuum filled waveguide and a muscovite mica disk $\left(0.1 \mathrm{~mm}\right.$ thickness, $\left.\varepsilon_{\mathrm{r}} \approx 7\right)$ as vacuum barrier, reflections are on the order of $-5 \mathrm{~dB}$ in $W$-band. For the Doppler reflectometer in ASDEX Upgrade tokamak, a bistatic setup using two phased-array antennas like [4], [5] (one for transmission, one for reception) is employed to mitigate the reflections from the vacuum barrier. However, monostatic antenna configurations (same antenna for transmission and reception) offer some advantages concerning alignment of their beam with the plasma, but at the expense of inherent reflection/leakage of the transmit power into the receiver from the single vacuum barrier - which can reduce the receiver sensitivity, or even cause saturation. Hence, it is important to minimize such reflections were possible.

There are several ways to reduce the reflectivity of vacuum barriers while still obtaining low leakage rates. Multilayer windows with quarter-wave spacing cancel the reflections by destructive interference and can provide low reflection within a rather limited relative bandwidth (on the order of $10 \%$ to $15 \%$ in $W$-band, which ranges from $75 \mathrm{GHz}$ to $110 \mathrm{GHz}$ ) [6], [7]. Brewster windows are built with oversized cylindrical hollow waveguides [8]-[10]. Here, the dielectric disk is inclined according to the Brewster angle. These windows can achieve a relative bandwidth of around $15 \%$ to $20 \%$ but installation space is rather large as a consequence of the oversized waveguides. Pill-box windows are made up from a cylindrical cavity with the dielectric disk placed in its center. These windows exhibit a relative bandwidth of around 15\% to $20 \%$, while consuming less installation space as compared to Brewster windows [11], [12]. Electrically thin windows can provide low reflection over the full band. However, at millimeter-wave frequencies their mechanical thickness is also very low and the gas leakage increases [6]. Windows with matching layers [13], [14] also reduce reflections but only within a limited bandwidth. Most frequently used window materials for millimeter-waves at the ASDEX Upgrade tokamak are muscovite mica and fused silica. Other plasma experiments use sapphire, chemical vapor deposition diamond [7], mylar, or epoxy [6].

In this work, we propose a vacuum window whose design is driven by three objectives. First, it should reduce window reflections within $32 \%$ relative bandwidth in the 


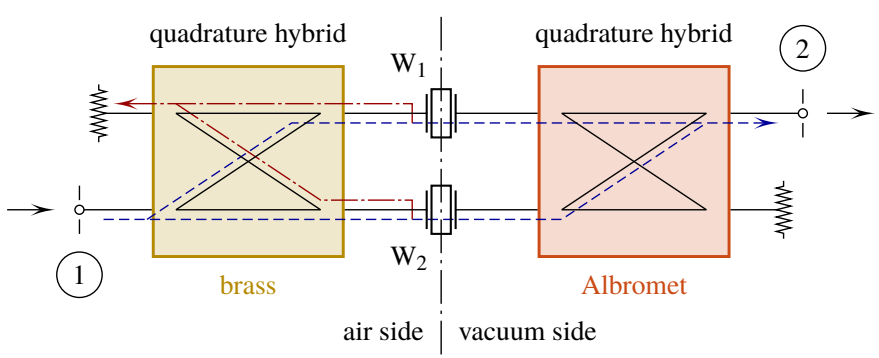

Fig. 1. Schematic of the proposed low reflectivity vacuum window. The principle is based on two identical mica vacuum windows $\mathrm{W}_{1}$ and $\mathrm{W}_{2}$ and a balanced hybrid lattice network. Shown are the paths of forward signal (dashed) and reflections (dash-dotted). Due to its symmetry this device behaves the same in the reverse direction.

$W$-band $(75 \mathrm{GHz}$ to $110 \mathrm{GHz})$. Second, a leak rate below $10^{-6} \mathrm{mbar} \mathrm{L} \mathrm{s}^{-1}$ should be preserved over an expected lifetime of approximately 10 years. During this period, it has to sustain the environmental conditions in the ASDEX Upgrade vessel (nuclear radiation, electromagnetic radiation ranging from infrared to hard X-ray, strong magnetic fields up to $2.8 \mathrm{~T}$, etc.) and vessel baking up to $150^{\circ} \mathrm{C}$. Last, the window design should support two waveguide channels fitting into one CF-35 flange, which is required for a bi-static Doppler reflectometer front-end. All current window designs are either too large or their operational bandwidth is too narrow. Consequently, a new design was necessary. The proposed low-reflectivity vacuum window is based on a balanced hybrid lattice network principle as shown in Fig. 1. This principle is well-known and has been used in the past in balanced amplifiers to improve the matching-bandwidth (e.g., [15]) and in interferometertype diplexing filters or balanced filter combiners (directional filter combiners) to separate reflections from input signals [16, p. 967], [17]. However, this principle has not yet been applied to vacuum barriers.

\section{LOW REFLECTIVITY WINDOW DESIGN}

The low reflectivity vacuum window is designed as a replacement for an existing vacuum window in the ASDEX Upgrade tokamak. The existing window consists of two WR 10 channels integrated in a ConFlat CF-35 vacuum flange. The vacuum barrier in each channel is a mica disk which is placed between two standard waveguide flanges. An O-ring (with very sparingly used vacuum grease) seals the connection and a leak rate below $10^{-6} \mathrm{mbar} \mathrm{L} \mathrm{s}^{-1}$ can be achieved. This design is similar to the single-disk window with a gasket ring in [3, p. 197].

The available space in the ConFlat CF-35 is approx. $\varnothing 35 \mathrm{~mm}$ with $20 \mathrm{~mm}$ axial length. Besides the vacuum and spatial constraints, there are also requirements due to strong magnetic fields and neutron radiation. These further restrict the material selection. For installation in the ASDEX Upgrade vessel, e.g., stainless steel 1.4301 or Albromet W-200 are allowed. The schematic of a single channel of the proposed vacuum window is shown in Fig. 1. The basic building blocks are two hybrid junctions, the vacuum barrier (two mica disks for this design), and two absorbers which dissipate the reflections from the mica disks.

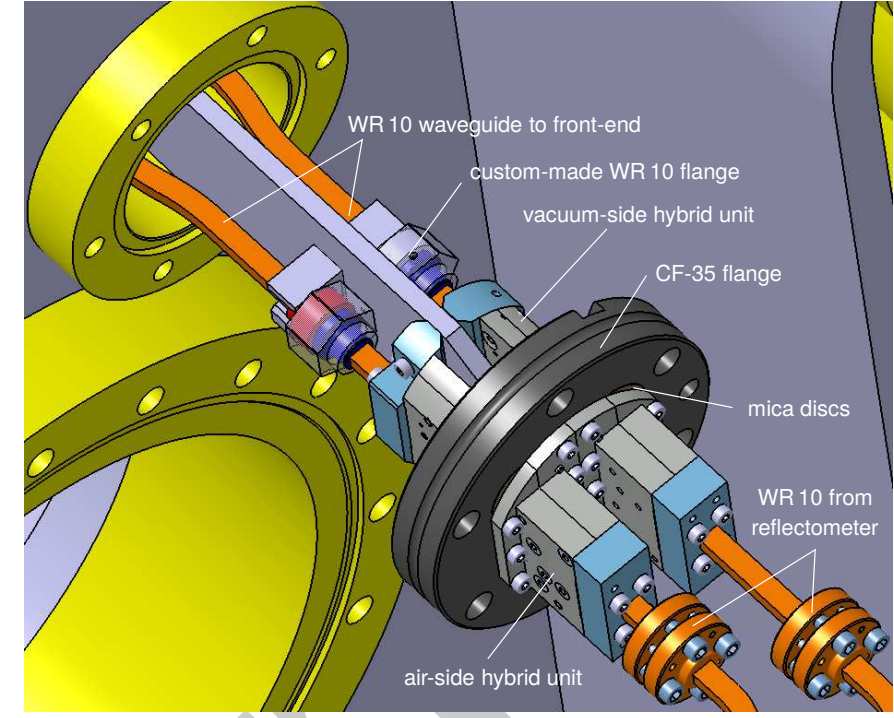

Fig. 2. Installation of the vacuum window in ASDEX Upgrade tokamak, Sector 11. Shielding of the CF-35 tube is hidden such that the interior is visible.

The principle can be explained as follows. A wave that is incident at port 1 is divided into two equal-power waves towards the vacuum windows $\mathrm{W}_{1}$ and $\mathrm{W}_{2}$. The signal at $\mathrm{W}_{1}$ has a $90^{\circ}$ phase offset relative to the signal at $\mathrm{W}_{2}$ due to the hybrid junction. At the windows, reflections occur because of the dielectric discontinuity and the waveguide surface current break. The remaining power is either transmitted through the windows or gets dissipated by dielectric loss. The reflected waves are directed back to the hybrid junction and again, the individual waves are equally split in terms of power. At port 1, the reflected waves interfere destructively. At the absorbing port, the individual reflections add up coherently. In brief, the hybrid junction directs the reflection from the vacuum barrier to the absorber. The same principle applies to the transmitted waves. They sum up coherently at port 2 because they experience equal $90^{\circ}$ phase shifts. At the absorbing port they appear with $180^{\circ}$ phase difference and none of the transmitted power is delivered there. To realize the vacuum window as sketched in Fig. 1, three individual elements are required: the hybrid junction in WR 10 waveguide technology, the absorber and the mica vacuum windows. The design of each individual component and the assembly of the window are described in the following sections. See Fig. 2, for the installation of the window in ASDEX Upgrade tokamak, Sector 11 . The hybrid junction and the absorber are combined in the hybrid units (one for each side of a window). Custom made WR 10 waveguide flanges connect the vacuum window to the pre-existing waveguides. The hexagonal waveguide flange is required for installation of the vacuum window in the ASDEX Upgrade vessel, due to rather limited accessibility of the CF-35 port.

\section{A. Quadrature Hybrid Junctions}

The quadrature hybrid junctions are realized as branchguide couplers, see Fig. 3. These allow for broadband operation covering the entire waveguide band [18]. Furthermore, 
their all-metal and structural shape can be fabricated by milling. The employed branch-guide coupler consists of seven branch-guides that interconnect two adjacent WR 10 waveguides (main guides). Its configuration is based on a design by Reed [19] which retains constant characteristic impedance of the main guides. Here, this design has been optimized to cover the frequency range from $75 \mathrm{GHz}$ to $105 \mathrm{GHz}$. The final design used in this device is identical to the one presented and discussed in [20]. The length of the branch-guides is approximately a quarter wavelength of the guided wave. Their height is adjusted so as to obtain an egual power split and minimum port reflection. In the final design the branchguides are $0.81 \mathrm{~mm}$ in length and the spacing between their centerlines is $1.07 \mathrm{~mm}$. The branch-guide height is $0.200 \mathrm{~mm}$ for the outer two branches and $0.340 \mathrm{~mm}$ for the inner five branches. The bandwidth is restricted by the number of slots which is limited by the minimum practical branch height. More branches allow for larger bandwidth. In our case the minimal realizable branch height is $0.200 \mathrm{~mm}$, which is given by the thinnest available milling tool with a milling depth of $1.27 \mathrm{~mm}$ (half-width of WR 10 waveguide). The performance of the branch-guide coupler might be improved by employing a design that also varies the main-guide impedance in addition to the branch-guide impedances [21].

\section{B. Isolated Port Terminations}

The isolated port terminations are based on a smooth and continuous transition of the guided wave field from the rectangular hollow waveguide into absorbing dielectric material, similar to the design presented in [22]. The absorbing material is a non-porously caked silicon carbide (Ekasic ${ }^{\circledR} F$ by ESK Ceramics GmbH \& Co. KG, 87405 Kempten, Germany) with expected $\varepsilon_{\mathrm{r}} \approx 10$ and $\tan \delta \approx 0.1$ (we did not characterize it in $W$-band). It does not outgas (i.e., measured leak rate below $2 \times 10^{-10} \mathrm{mbar} \mathrm{L} \mathrm{s}^{-1}$ of a $24.7 \mathrm{~mm} \times 6.15 \mathrm{~mm} \times 85 \mathrm{~mm}$ material sample) and it is heat resistant up to more than $1500{ }^{\circ} \mathrm{C}$. Silicon carbide ( $\left.\mathrm{SiC}\right)$ is extremely hard. Thus, it is difficult to machine and diamond tools have to be used. Normally, it is shaped and formed by a sintering process. However, the latter requires a special mold which is expensive and, therefore, not cost-efficient at low lot sizes. Consequently, we sliced an available material sample into rectangular wafers. A smooth transition of the guided wave into the $\mathrm{SiC}$ wafer is realized by a linear taper. The taper is formed by inclining the rectangular hollow waveguide relative to the $\mathrm{SiC}$ wafer's edge.

For the vacuum window, the $\mathrm{SiC}$ wafer measures $12 \mathrm{~mm}$ in length, $6.15 \mathrm{~mm}$ in width, and $2.0 \mathrm{~mm}$ in height. It is inclined by $31.5^{\circ}$ relative to the WR 10 waveguide to realize the tapered transition, see Fig. 3. A $148.5^{\circ}$ bend connects the absorber to the branch-guide coupler. The $\mathrm{SiC}$ wafer is fixed in position by a tiny leaf spring which also compensates for thermal elongation. This design is mainly driven by the space restrictions inside the $\mathrm{CF}-35$ flange. A simulation from CST Microwave Studio (version 2017.2) of the absorber in Fig. 3 including the $148.5^{\circ}$ bend is given in Fig. 4. The reflectivity is below $-17 \mathrm{~dB}$, which is considered sufficient because of

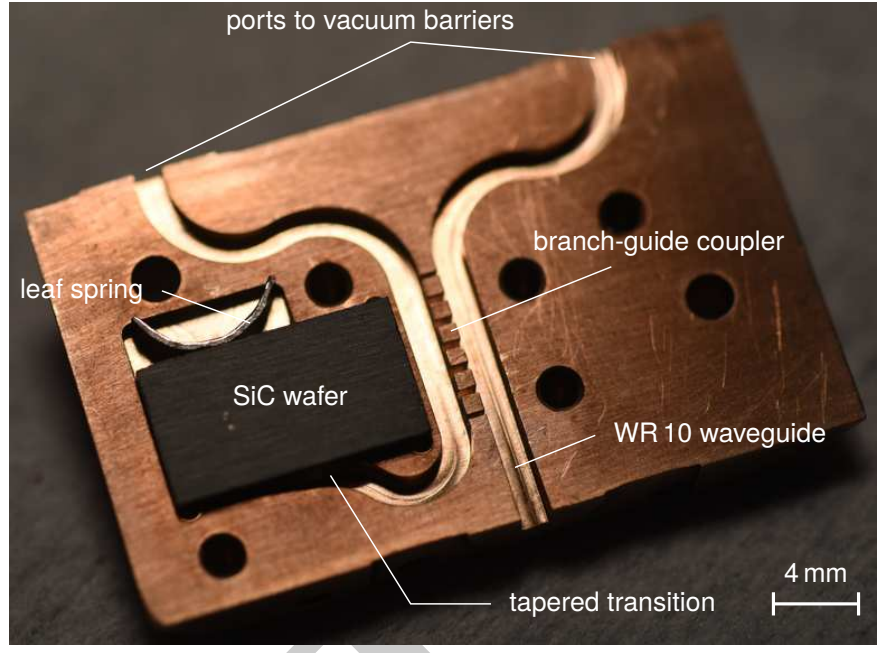

Fig. 3. Detailed view of silicon carbide absorber and branch-guide coupler in one half of the milled split block. A bent flat leaf spring is used to keep the absorber in its correct position.

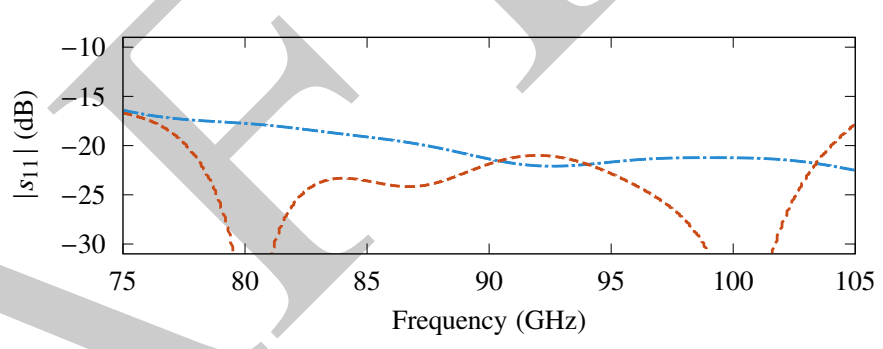

Fig. 4. Simulation results of the absorber in the proposed vacuum window (dash-dotted), as in Fig. 3, including the $148.5^{\circ}$ bend. Simulated reflection of the branch-guide coupler with short-circuited ports to the vacuum barrier and a perfect absorber (dashed). Conductivity of waveguide walls is $12.5 \times 10^{6} \mathrm{~S} \mathrm{~m}^{-1}, \mathrm{SiC}$ wafer is specified by $\varepsilon_{\mathrm{r}}=10$ and $\tan \delta=0.1$.

two reasons. First, the major part of the reflection from the absorber is transmitted through the hybrid lattice network to the absorber on the other side of the windows and dissipated there. Second, the part of the reflections that is itself reflected from the windows and directed to the input port appears at a similar level as the reflections of the hybrid junction itself with short-circuited ports to the vacuum barrier (see Fig. 4). The measurement with short circuited ports allow the influence of quadrature errors, finite directivity, and mismatch of the branch-guide coupler to be seen. It does not consider the non-identical and slightly weaker reflection coefficients of the windows. If more installation space is available, the reflections of the absorber can be reduced by a smoother/longer tapered transition of the WR 10 waveguide into the $\mathrm{SiC}$ wafer.

\section{Vacuum Barrier}

The vacuum barrier is realized by a muscovite mica disk, which is sealed by an O-ring and vacuum grease, see Fig. 6. The mica disk has openings and cutouts for flange alignment pins and screws. The mica windows are laser-cut from a muscovite mica sheet. The mica sheet (mica special plates by Richard Jahre GmbH, 26388 Wilhelmshaven, Germany) has a thickness of $0.1 \mathrm{~mm}$ and its relative dielectric constant is specified in the datasheet as $\varepsilon_{\mathrm{r}}=6 \ldots 8$ with a loss 
tangent of $\tan \delta=1 \times 10^{-4} \ldots 8 \times 10^{-4}$. Fused silica would be preferable because of its lower dielectric constant and thereby lower reflection/insertion loss, but mismatch of the thermal expansion coefficient between fused silica and metal implies a significant risk of breaking. The mechanical structure could be designed to compensate this effect, but the focus of this prototype was more on the proof of concept with an established vacuum barrier rather than on achieving the best possible results.

A simulation using CST Microwave Studio (version 2017.2) as well as measurements of a single mica disk in-between two anti-cocking WR 10 waveguide flanges are given in Fig. 5. The measurements were carried out by means of an HP8510 C vector network analyzer and thru-reflect-line calibration. The reference planes are located at the waveguide to mica interface. In the simulation, a $1 \mathrm{~mm}$ waveguide segment is added at each side of the mica disk. The measurements were carried out for two different mica disks. In the simulation the thickness of the mica disk is $0.1 \mathrm{~mm}$ and $\varepsilon_{\mathrm{r}}=7$ is assumed. The transmission through the mica disks is around $-3 \mathrm{~dB}$ to $-2 \mathrm{~dB}$ and reflections are in the range from $-6 \mathrm{~dB}$ to $-4 \mathrm{~dB}$. The simulated window shows spurious resonances in all S-parameters. However, these do not coincide with those of the measured windows. Most likely, the observed frequency shift is due to manufacturing tolerances of the mica disk and possible misalignment of the waveguide flanges during the measurement. The overall characteristics of the measured S-parameters are similar for both mica disks. Yet, there are small differences - presumably due to the thickness tolerance of the muscovite mica sheet, which is $\pm 0.01 \mathrm{~mm}$. As a consequence, the cancellation of reflections at the input port of the hybrid unit is not perfect. The remaining reflection amplitude is estimated by subtracting both complex valued reflection amplitudes (weighted with $1 / 2$ due to passing the hybrid junction twice) from the individual mica disks, see bottom of Fig. 5. The estimated reflectivity is below $-25 \mathrm{~dB}$ over the design frequency range with the exception of two peaks, but always smaller than $-20 \mathrm{~dB}$. This is considered sufficient. Crosstalk between two adjacent waveguide channels through the mica disks has been analyzed by simulation which pointed to values below $-58 \mathrm{~dB}$ over the frequency range $75 \mathrm{GHz}$ to $105 \mathrm{GHz}$.

To make a good vacuum seal, an O-ring is placed in a groove around the waveguide cross-section, see Fig. 8 for a technical drawing of the flange. The mica disk is placed on top of it. The pressure of the adjoining waveguide flange, see Fig. 7, on the mica disk and the O-ring accomplish the vacuum barrier. This design is rather insensitive to thermal elongation as the mica is relatively flexible (in contrast to fused silica). At the ASDEX Upgrade tokamak, the expected lifetime of such a vacuum seal is 10 years. After 12 years, the probability of failure or leakage increases significantly due to aging of the O-ring from numerous vessel bake-out cycles up to $150{ }^{\circ} \mathrm{C}$. In exceptional cases, the maximum allowed leak rate in the ASDEX Upgrade tokamak is $10^{-6} \mathrm{mbar} \mathrm{L} \mathrm{s}^{-1}$. Four of these vacuum barriers are made in a single CF-35 flange, see Fig. 6. The WR 10 waveguides are spark-eroded, then the O-ring grooves and alignment pin holes are manufactured with

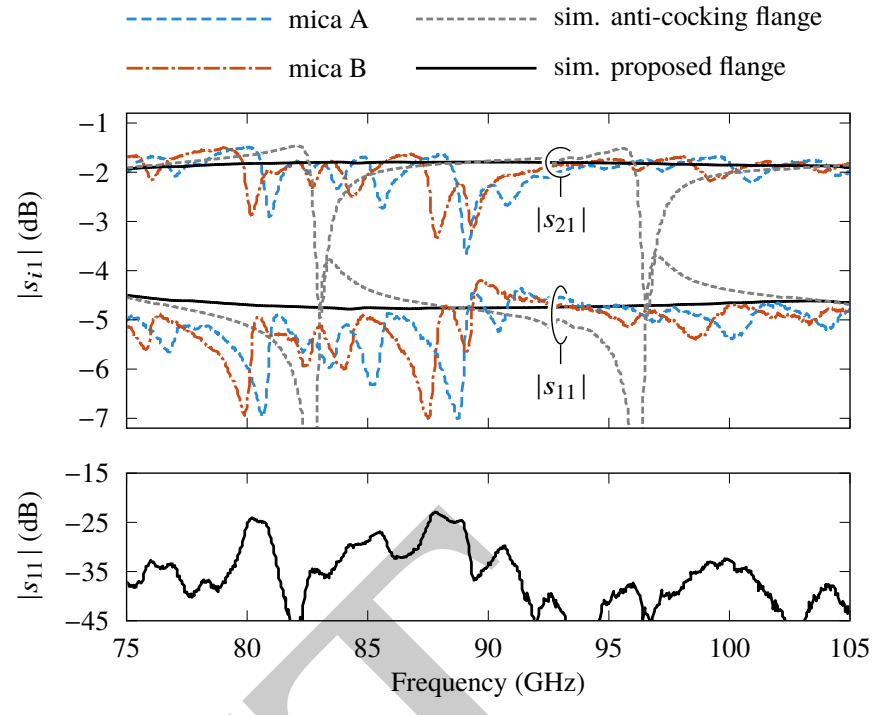

Fig. 5. Top: Simulation of a mica disk $\left(0.1 \mathrm{~mm}\right.$ thick, $\left.\varepsilon_{\mathrm{r}}=7\right)$ with a proposed waveguide flange (Figs. 7 and 8) and a WR 10 waveguide anti-cocking flange connection, as well as measurement results of two different mica disks placed between a WR 10 waveguide anti-cocking flange connection. The difference in the measured S-parameters is due to the thickness tolerances of the mica $( \pm 0.01 \mathrm{~mm})$. Bottom: Remaining amplitude after subtracting complex measurement results (destructive interference).

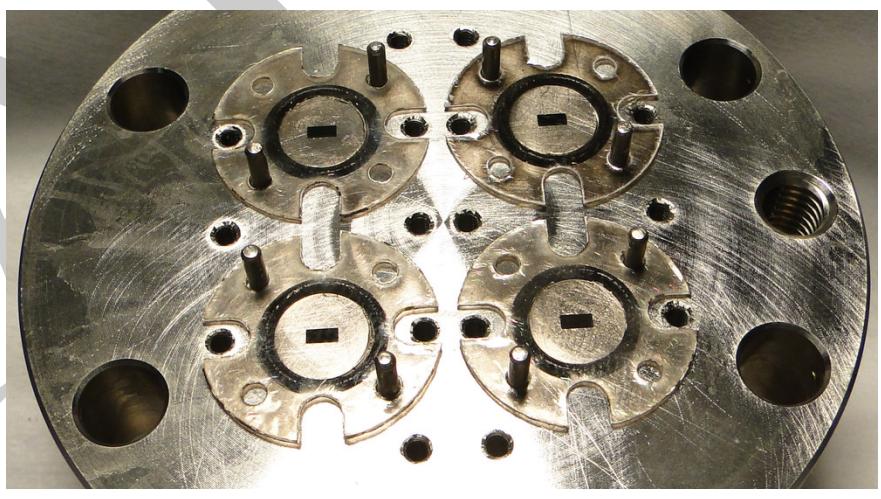

Fig. 6. CF-35 vacuum flange with four spark-eroded WR 10 waveguide feedthroughs and required fixture holes. Each feedthrough is surrounded by an O-ring in a groove and capped by a laser-cut mica disk.

aid of a jig borer.

As can be seen in Fig. 5, cylindrical waves can exist inside the mica disk and lead to resonant peaks in the insertion loss. To suppress this effect, a choke groove is milled around the waveguide opening of the flange of the hybrid unit introducing a discontinuity in radial direction of the dielectric disk (see Fig. 7 and Fig. 8). Thereby, spurious resonances are shifted out of the operating frequency range. The choke groove has a depth of $0.2 \mathrm{~mm}$ and a width of $0.5 \mathrm{~mm}$ (approximately a quarter wavelength in the material mix of $0.1 \mathrm{~mm}$ muscovite mica and $0.2 \mathrm{~mm}$ air). The wall between the rectangular hollow waveguide and the choke groove is $0.25 \mathrm{~mm}$ (also roughly a quarter wave in the muscovite mica). Simulation results of the proposed flange can be seen in Fig. 5. The manufactured choke groove is not perfect as it appears slightly unsymmetrical with respect to the waveguide cross-section, but its functionality is not significantly affected by this (see section III). 


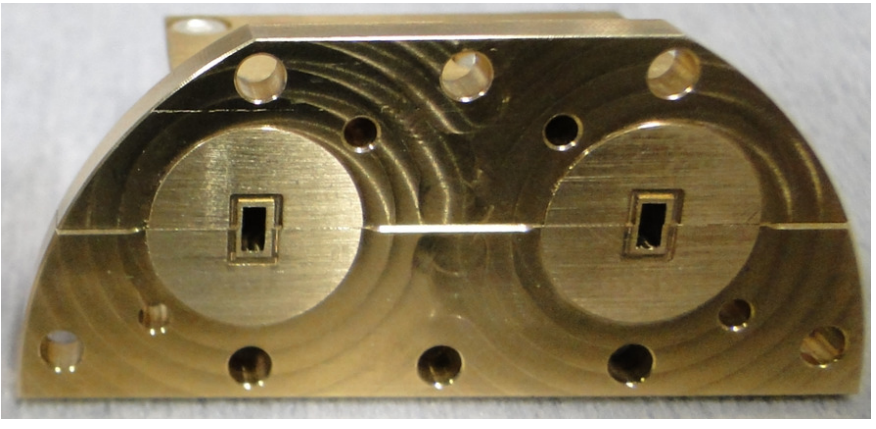

Fig. 7. Flange face of an ex-vessel hybrid unit, large circular face dedicated to clamp mica disk and O-ring. The choke suppresses resonant modes within the mica disk, but could not be manufactured perfectly symmetric.
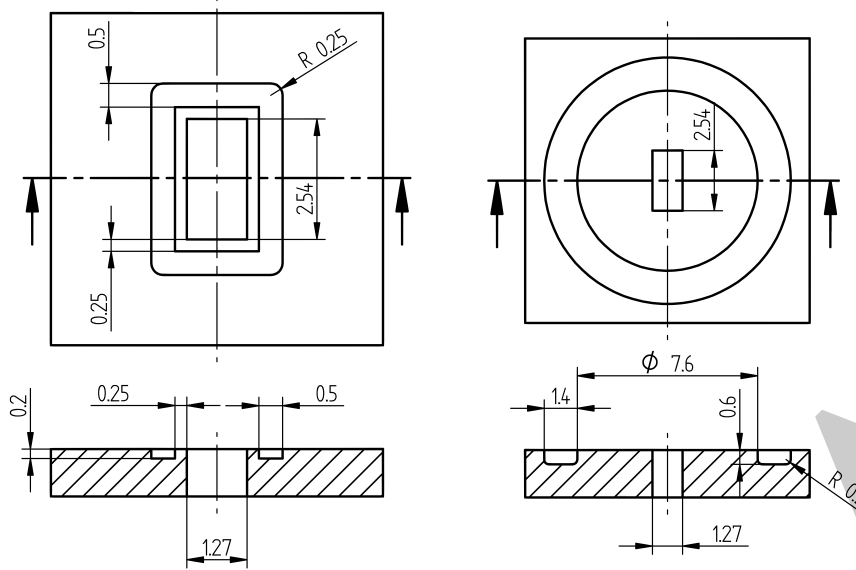

Fig. 8. Drawings and dimensions (in $\mathrm{mm}$ ) of the flange faces that connect the CF-35 flange with the air-side hybrid unit. In the air-side hybrid unit flange, there is a choke groove to suppress resonant modes within the mica disk (left) whereas the opposing waveguide flange in the CF-35 flange includes an Oring for vacuum sealing (right), compare Figs. 6 and 7 (different in scale for a better contrast of details).

\section{Window Assembly}

The hybrid coupler and the absorber are made in the same split block and arranged such that they fit into the CF-35 flange, see Fig. 3. All in-vessel split-block parts are fabricated from Albromet W-200 (EN CW 112 C). This material is a high-conductive and non-magnetic copper alloy which is suited for in-vessel installation in ASDEX Upgrade tokamak (nominal conductivity: $22 \times 10^{6} \mathrm{~S} \mathrm{~m}^{-1}$ ). Alternative materials for in-vessel use are stainless steal 1.4301 (nominal conductivity: $1.37 \times 10^{6} \mathrm{~S} \mathrm{~m}^{-1}$ ) or almost pure aluminum AL99.5 (nominal conductivity: $35 \times 10^{6} \mathrm{~S} \mathrm{~m}^{-1}$ ). Stainless steel has a lower conductivity as compared to Albromet W-200, and aluminum AL99.5 is considerably softer so that the small slots might be smeared during manufacturing. The ex-vessel parts (no vacuum requirements) are fabricated from brass CuZn37 (nominal conductivity: $15.2 \times 10^{6} \mathrm{~S} \mathrm{~m}^{-1}$ ) due to its considerably better machinability as compared to Albromet W-200.

Two sets of parts were made. All parts with visible flaws and deficiencies were arranged to build up one channel (marked as channel A) while all parts without such deficiencies were used for the second channel (marked as channel B). The objective was to have at least one channel in good working order.

The vacuum side of the assembled window can be seen in Fig. 9 and a side view is shown in Fig. 10. The CF-35 flange is mounted to the ASDEX Upgrade vessel, as shown in Fig. 2. Custom made flanges connect the window to preexisting waveguides.

\section{Performance Results}

The vacuum window was tested with a helium leak detector. The first test was at room temperature where the measured helium leakage rate was $1.5 \times 10^{-9} \mathrm{mbar} \mathrm{L} \mathrm{s}^{-1}$. Next, it was heated to $150^{\circ} \mathrm{C}$ - the bake-out temperature of the ASDEX Upgrade vessel - where the leakage rate rose up to $5.6 \times 10^{-7} \mathrm{mbar} \mathrm{L} \mathrm{s}^{-1}$. After cooling down to room temperature again a leakage rate of $8 \times 10^{-10} \mathrm{mbar} \mathrm{L} \mathrm{s}^{-1}$ was observed. With these results, it is certified for use in the ASDEX Upgrade tokamak experiment.

A finite-difference time domain simulation of the proposed vacuum window has been conducted in CST Microwave Studio (version 2016.2). The hybrid units (consisting of a branch-guide coupler and an absorber), the CF-35 flange, the O-rings, and the mica disks where included in the simulation. The resulting transmission and reflection are given in Fig. 11 for the direction air to vacuum and in Fig. 12 for the direction vacuum to air. The reflection from the vacuum barrier is reduced to below $-15 \mathrm{~dB}$ (from about $-4 \mathrm{~dB}$ of the mica disk alone, see Fig. 5). The transmission is slightly worse (around $-2.8 \mathrm{~dB}$ ) as compared to the mica disk (around $-1.9 \mathrm{~dB}$ ) due to the Ohmic losses in the hybrid lattice network. S-parameter measurements were carried out on the HP $8510 \mathrm{C}$ vector network analyzer with millimeter-wave extensions and thru-reflect-line calibration and the results are shown in in Fig. 11 and Fig. 12. The measured transmission is lowered by approximately $1.7 \mathrm{~dB}$ compared to the simulated transmission. This is due to additional $330 \mathrm{~mm}$ copper waveguide with approximately $1.1 \mathrm{~dB}$ attenuation (from $150 \mathrm{~mm}$ reference waveguide with $0.5 \mathrm{~dB}$ attenuation) and three custom made waveguide flanges that were needed in the measurement setup, but could not be included in the calibrated section because of non-standard waveguide flanges. The measured reflectivity remains below $-15 \mathrm{~dB}$ over the entire design range from $75 \mathrm{GHz}$ to $105 \mathrm{GHz}$.

In the simulation and in the measurements, the reflectivity of the proposed window exhibits a ripple with relatively high peak-to-valley ratio. A possible cause is interference between branch-guide coupler reflections and reflections from the absorber that get re-reflected from the windows and are directed to the input port. Both reflections appear there at approximately similar amplitude levels around $-20 \mathrm{~dB}$. The slope of the $\left|s_{22}\right|$ curve differs from that of $\left|s_{11}\right|$ because the realized structure is not strictly symmetric with respect to the plane of the mica disks. The mica disks are assembled on the air side of the CF-35 flange. Due to the thickness of the CF-35 flange, the electrical propagation lengths of the individual reflected waves from the absorbers are different. As a consequence, the interference of these reflections with those from the branch-guide coupler have a different frequency 


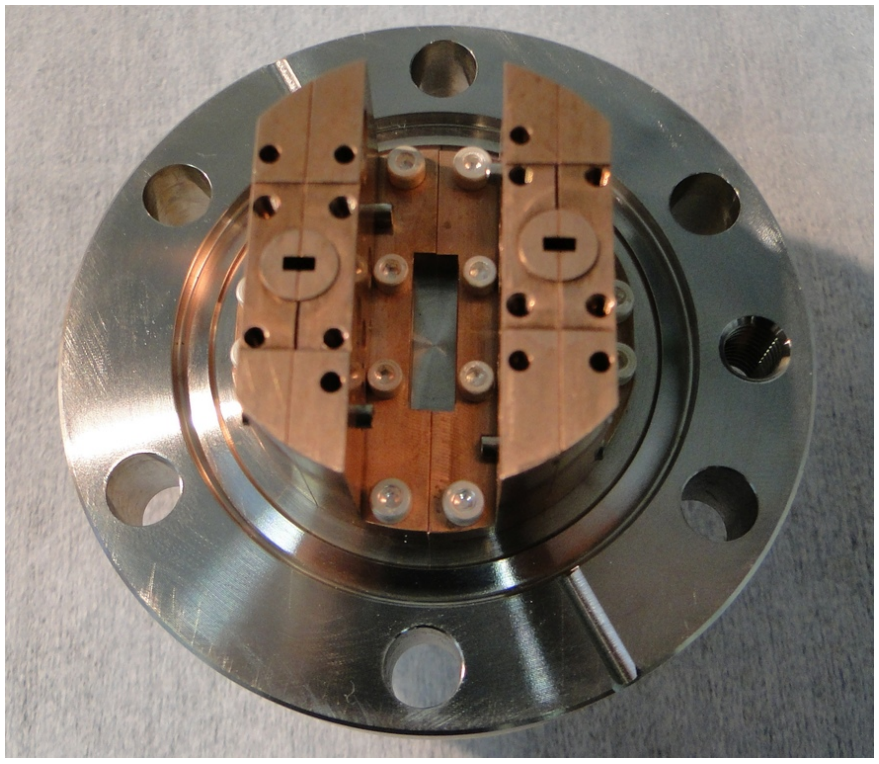

Fig. 9. Photo of the completely assembled vacuum window, as seen from the vacuum side (in-vessel parts). In the front there are the two waveguide flanges for channels A and B. Split-block parts on the vacuum side are fabricated from Albromet W-200 while those on air side are fabricated from brass.

dependency on either side of the vacuum barrier. While this does not impair the functioning of the balanced circuit, it still results in an asymmetric scattering matrix $\left(s_{11} \neq s_{22}\right)$.

The difference in the window reflection coefficients has no effect on the reflection coefficients of the complete assembly, as simulated and measured data show a good agreement, see Fig. 11 and Fig. 12. This was also expected from the estimate in Fig. 5, where the resulting reflection is far below $-20 \mathrm{~dB}$ and can be neglected with respect to the branch-guide coupler and absorber reflections.

Channel A, which contains all the poor quality parts (see section II-D), shows a local peak in $\left|s_{11}\right|$ at around $95 \mathrm{GHz}$. Corresponding features are seen in $\left|s_{21}\right|$ and $\left|s_{12}\right|$, but not in $\left|s_{22}\right|$. This phenomenon is an indication of a defect at the air-side hybrid junction. However, we did not re-open the assembly to investigate this effect as channel B works as desired and the vacuum test would have to be repeated.

\section{CONCLUSION}

A low-reflectivity vacuum window has been realized in WR 10 technology. It is based on a balanced hybrid lattice network. Broadband operation is achieved by branch-guide hybrid couplers. The reflectivity is mostly below $-20 \mathrm{~dB}$ in the frequency range from $75 \mathrm{GHz}$ to $105 \mathrm{GHz}$. The measured maximum reflectivity is $-15 \mathrm{~dB}$ for a prototype built from selected parts. A helium leakage rate of $8 \times 10^{-10} \mathrm{mbar} \mathrm{L} \mathrm{s}^{-1}$ was achieved. The window has been installed in the ASDEX Upgrade tokamak and may enable mono-static operation of future Doppler reflectometer front-ends. Compared to state-of-the-art window designs, low reflectivity over almost a full waveguide band can be achieved while consuming only slightly more installation space than a simple window consisting of one dielectric sheet between two waveguide flanges. The proposed design may be combined with other window technologies (e.g.,

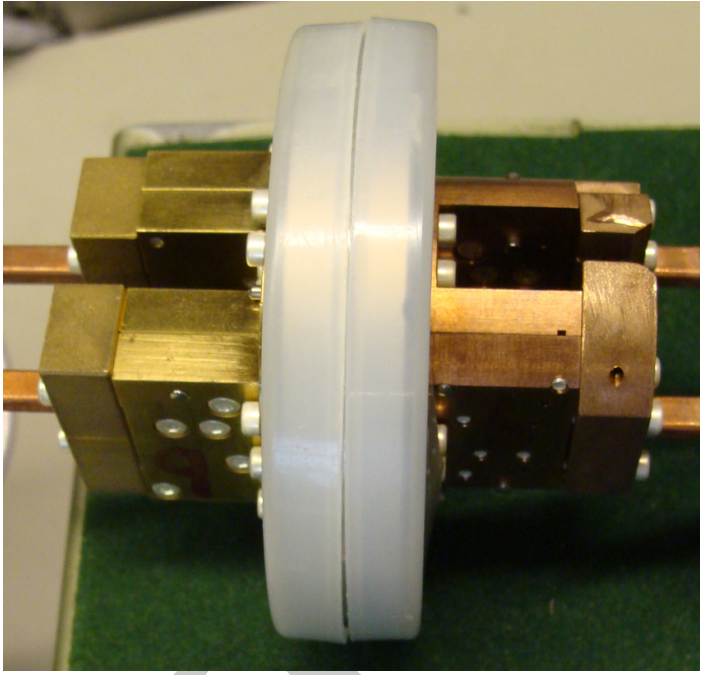

Fig. 10. Side view of the window. CF-35 flange in plastic covers. Left: air-side, right: vacuum-side. Custom made flanges and waveguide sections connect the window interface to the measurement reference plane.

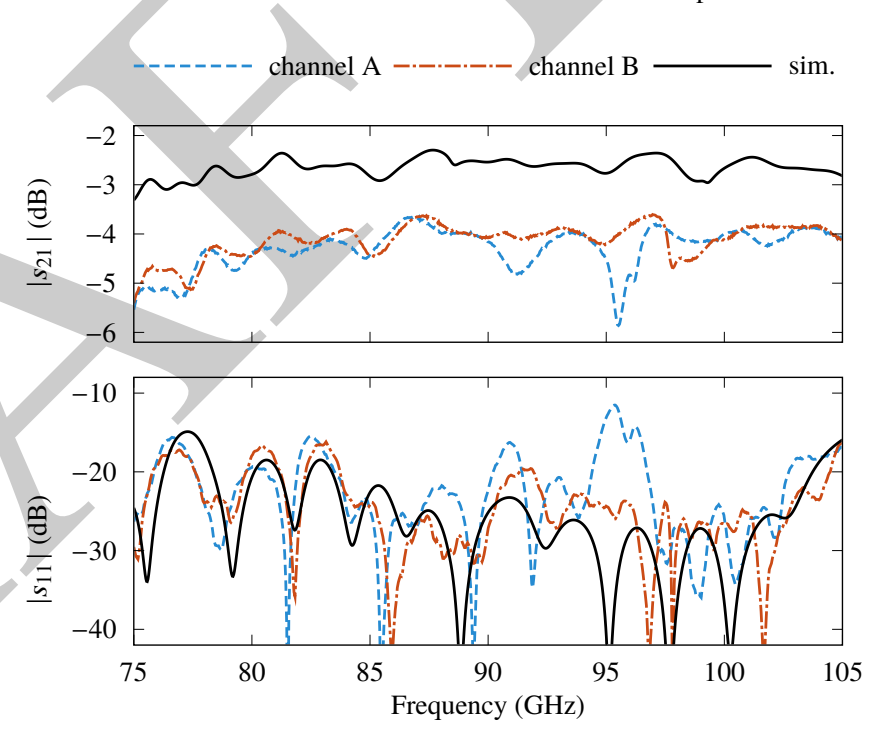

Fig. 11. Simulated and measured S-parameters of the two separate channels of this vacuum window (direction air-vacuum). The measured assembly includes a $330 \mathrm{~mm}$ copper waveguide section with approximately $1.1 \mathrm{~dB}$ attenuation and three custom flanges. The simulated S-parameters are relative to the flange faces of the hybrid units.

multilayer windows), or made from a material with lower dielectric constant than mica (e.g., fused silica) to reduce the insertion loss and relax the requirements on the reflectivity of the absorber. If more installation space is available, the mainguide height of the branch-guide coupler can be adjusted in order to further improve the bandwidth or electrical properties of the hybrid junction (e.g., [21]) and the tapered transition into the $\mathrm{SiC}$ wafer can be designed with a smaller inclination angle in order to reduce the reflections from the absorber.

\section{ACKNOWLEDGMENT}

The authors thank Johannes Friesen, Daniel Grossmann and the mechanical workshop of the ASDEX Upgrade tokamak experiment at IPP Garching for their support. 

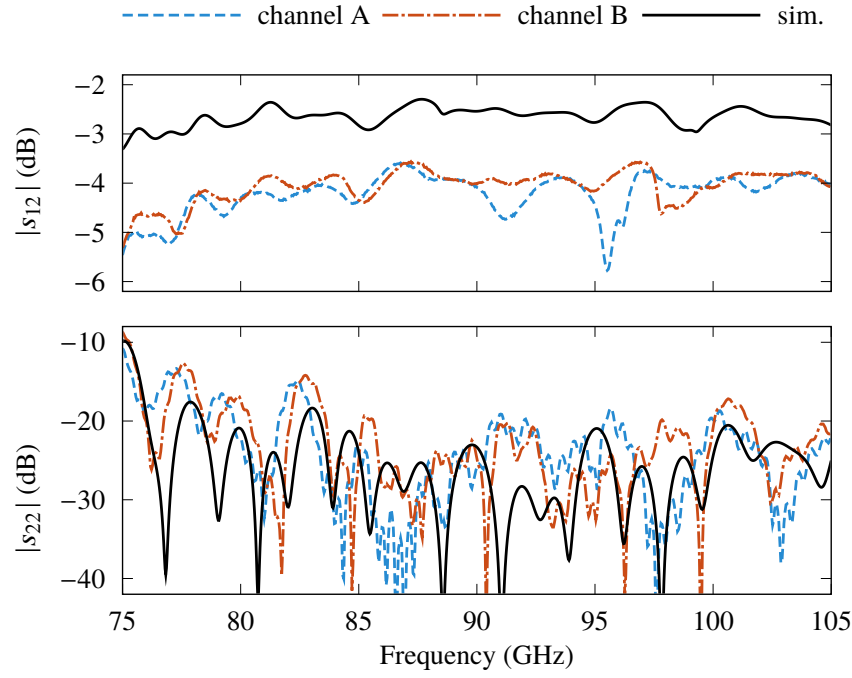

Fig. 12. Simulated and measured S-parameters of the two separate channels of this vacuum window (direction vacuum-air). The measured assembly includes a $330 \mathrm{~mm}$ copper waveguide section with approximately $1.1 \mathrm{~dB}$ attenuation and three custom flanges. The simulated S-parameters are relative to the flange faces of the hybrid units.

\section{REFERENCES}

[1] G. D. Conway, "Turbulence measurements in fusion plasmas," Plasma Phys. Controlled Fusion, vol. 50, no. 12, p. 124026, 2008.

[2] G. D. Conway, E. Poli, T. Happel, and the ASDEX Upgrade Team "Interaction of mean and oscillating plasma flows across confinement mode transitions," Plasma and Fusion Research, vol. 5, p. S2005, 2010

[3] H.-J. Hartfuss and T. Geist, Fusion Plasma Diagnostics with mm-Waves: An Introduction. Weinheim, Germany: Wiley-VCH, 2013.

[4] P. Rohmann, S. Wolf, W. Kasparek, B. Plaum, and J. Hesselbarth, "A 32element frequency-steered array antenna for reflectometry in W-band," in IEEE Int. Symp. Phased Array Systems and Technology, Waltham, MA, USA, October 2013, pp. 559-563.

[5] C. Koenen, U. Siart, T. F. Eibert, G. D. Conway, and U. Stroth, "Design of a millimeter-wave phased array antenna for Gaussian beam shaping and steering," in IEEE Int. Symp. Phased Array Systems and Technology, Waltham, MA, USA, October 2016.

[6] G. A. Ediss, N. Horner, F. Johnson, D. Koller, and A. R. Kerr, "WR10 waveguide vacuum feedthrough for the ALMA band-6 cartridge," NRAO ALMA memo 536, National Radio Astronomy Observatory, Charlottesville, VA, 2005.

[7] C. R. Donaldson, W. He, L. Zhang, and A. W. Cross, "A W-band multilayer microwave window for pulsed operation of gyro-devices," IEEE Microw. Wireless Compon. Lett., vol. 23, no. 5, pp. 237-239, May 2013.

[8] D. Wagner, W. Kasparek, M. Thumm, G. Gantenbein, and H. Zohm, "Transmission line design for broadband microwave diagnostic systems," in Diagnostics for Experimental Thermonuclear Fusion Reactors 2, P. E. Stott, G. Gorini, P. Prandoni, and E. Sindoni, Eds. New York: Springer US, 1998, ch. 16, pp. 161-170.

[9] G. Gantenbein et al., "First operation of a step-frequency tunable 1-MW gyrotron with a diamond brewster angle output window," IEEE Trans. Electron Devices, vol. 61, no. 6, pp. 1806-1811, June 2014.

[10] Y. Zhang et al., "Design and measurement of a W-band brewster window," IEEE Microw. Wireless Compon. Lett., vol. 25, no. 12, pp. 826-828, December 2015.

[11] H. Arai, N. Goto, Y. Ikeda, and T. Imai, "An analysis of a vacuum window for lower hybrid heating," IEEE Trans. Plasma Sci., vol. 14, no. 6, pp. 947-954, June 1986.

[12] S. Liu, "A RF window for broadband millimeter wave tubes," International Journal of Infrared and Millimeter Waves, vol. 17, no. 1, pp. 121-126, January 1996.

[13] M.-C. Lin, "A multilayer waveguide window for wide-bandwidth millimeter wave tubes," International Journal of Infrared and Millimeter Waves, vol. 28, no. 5, pp. 355-362, May 2007.

[14] D. Koller, A. R. Kerr, G. A. Ediss, and D. Boyd, "Design and fabrication of quartz vacuum windows with matching layers for millimeter- wave receivers," NRAO ALMA memo 377, National Radio Astronomy Observatory, Charlottesville, VA, 2001

[15] K. Kurokawa, "Design theory of balanced transistor amplifiers," The Bell System Technical Journal, vol. 44, no. 8, pp. 1675-1698, October 1965.

[16] G. Matthaei, L. Young, and E. Jones, Microwave Filters, ImpedanceMatching Networks, and Coupling Structures, ser. Artech Microwave Library. Norwood, MA: Artech House, 1980.

[17] T. Geist and U. Siart, "Diplexing millimeter waves," International Journal of Infrared and Millimeter Waves, vol. 19, no. 7, pp. 957-962, July 1998.

[18] S. B. Cohn and R. Levy, "History of microwave passive components with particular attention to directional couplers," IEEE Trans. Microw. Theory Techn., vol. 32, no. 9, pp. 1046-1054, 1984.

[19] J. Reed, "The multiple branch waveguide coupler," IRE Trans. Microw. Theory Techn., vol. 6, no. 4, pp. 398-403, April 1958.

[20] C. Koenen, U. Siart, T. F. Eibert, and G. D. Conway, "A self-aligning cylindrical sliding short plunger for millimeter-wave rectangular waveguides and its application in a reflection-type phase shifter," IEEE Trans. Microw. Theory Techn., vol. 65, no. 2, pp. 449-458, February 2017.

[21] R. Levy, "Zolotarey branch-guide couplers," IEEE Trans. Microw. Theory Techn., vol. 21, no. 2, pp. 95-99, February 1973.

[22] F. P. Mena and A. M. Baryshev, "Design and simulation of a waveguide load for ALMA-band 9," NRAO ALMA memo 513, National Radio Astronomy Observatory, Charlottesville, VA, 2005. 